\title{
ON THE DEGREE OF AN INDECOMPOSABLE REPRESENTATION OF A FINITE GROUP
}

\author{
GERALD H. CLIFF
}

(Received 24 August 1978)

Communicated by D. E. Taylor

\begin{abstract}
Let $k$ be an algebraically closed field of characteristic $p$, and $G$ a finite group. Let $M$ be an indecomposable $k G$-module with vertex $V$ and source $X$, and let $P$ be a Sylow $p$-subgroup of $G$ containing $V$. Theorem: If $\operatorname{dim}_{k} X$ is prime to $p$ and if $N_{G}(V)$ is $p$-solvable, then the $p$-part of $\operatorname{dim}_{k} M$ equals $[P: V] ; \operatorname{dim}_{k} X$ is prime to $p$ if $V$ is cyclic.
\end{abstract}

1980 Mathematics subject classification (Amer. Math. Soc.): 20 C 20.

Let $k$ be an algebraically closed field of characteristic $p$. Suppose that $M$ is an indecomposable $k G$-module, where $G$ is a finite group. If $V$ is a vertex of $M$ and $V$ is contained in a Sylow $p$-subgroup $P$ of $G$, then Green (1959) has shown that $[P: V]$ divides the dimension of $M$ over $k$. In this note, we show that $[P: V]$ is equal to the $p$-part of $\operatorname{dim}_{k} M$ if a source of $M$ has dimension prime to $p$ and if $N_{G}(V)$ is $p$-solvable. We may therefore determine the $p$-part of $\operatorname{dim}_{k} M$ if $V$ is cyclic and $N_{G}(V)$ is $p$-solvable.

Notation. All modules are finitely generated left modules. We understand $L \mid M$ to mean that $L$ is isomorphic to a direct summand of $M$. If $M$ is a $k H$-module, where $H$ is a subgroup of $G$, then $M^{G}$ denotes the induced module. For a positive integer $n$, we denote the $p$-part of $n$ by $n_{p}$.

THEOREM. Let $G$ be a finite group and let $M$ be an indecomposable $k G$-module with vertex $V$ and source $X$. If $p \nmid \operatorname{dim}_{k} X$, and $N_{G}(V)$ is p-solvable, then $\left(\operatorname{dim}_{k} M\right)_{p}=[G: V]_{p}$. 
Proof. We first assume that $V$ is normal in $G$, so $G=N_{G}(V)$ is $p$-solvable. Let $T$ be the inertia group of $X$ in $G$ :

$$
T=\{g \in G: g \otimes X \cong X\}
$$

According to the results of Conlon (1964) and Tucker (1965), there exists a twisted group algebra $A$ on $T / V$ over $k$ with the following property: if $A=\sum_{i=1}^{n} U_{i}$ is a decomposition of $A$ into a direct sum of indecomposable left ideals, then there is a decomposition $X^{G}=\sum_{i=1}^{*} M_{i}$ into indecomposable $k G$-submodules of $X^{G}$ such that

$$
\operatorname{dim}_{k} M_{i}=\left(\operatorname{dim}_{k} X\right)\left(\operatorname{dim}_{k} U_{i}\right)[G: T], \quad 1 \leqslant i \leqslant n
$$

(The algebra $A$ is a homomorphic image of $\operatorname{End}_{k T}\left(X^{T}\right)$.) Since $M$ has source $X$, then $M \mid X^{G}$, so $M$ is isomorphic to one of the $U_{i}$. We show that $\left(\operatorname{dim}_{k} U_{i}\right)_{p}=[T: V]_{p}$.

We prove that if $U$ is an indecomposable summand of a twisted group algebra on a finite $p$-solvable group $H$ over $k$, then $\left(\operatorname{dim}_{k} U\right)_{p}=|H|_{p}$. We use induction on $|H|$. Denote the twisted group algebra by $(k H)_{\alpha}$, where $\alpha$ is the factor set on $H$ of the algebra. Let $R$ be a normal subgroup of $H$ such that $H / R$ is a $p$-group or a $p^{\prime}$-group; let $(k R)_{\alpha}$ be the twisted group algebra on $R$ whose factor set is the restriction of $\alpha$ to $R$. Then there is an indecomposable $(k R)_{\alpha}$-module $W$ such that $U \mid W^{H}=W \otimes_{(k R)_{\alpha}}(k H)_{\alpha}$. We apply the results of Conlon (1964) to $W^{H}$. Let $S$ be the inertial group of $W$ in $H$; then there is a twisted group algebra $A^{\prime}$ on $S / R$ over $k$, with an indecomposable summand $U^{\prime}$, such that

$$
\operatorname{dim}_{k} U=\left(\operatorname{dim}_{k} W\right)\left(\operatorname{dim}_{k} U^{\prime}\right)[H: S]
$$

By induction on $|H|$, we have $\left(\operatorname{dim}_{k} W\right)_{p}=|R|_{p}$. If $H / R$ is a $p$-group, then so is $S / R$, hence $A^{\prime}$ is isomorphic to the (untwisted) group algebra $k(S / R)$ and is therefore indecomposable. Thus $U^{\prime}=k(S / R)$, so $\operatorname{dim}_{k} U^{\prime}=[S: R]$. We have, from (2),

$$
\left(\operatorname{dim}_{k} U\right)_{p}=|R|_{p}[S: R]_{p}[H: S]_{p}=|H|_{p}
$$

If $H / R$ is a $p^{\prime}$-group, then $A^{\prime}$ is a twisted group algebra on a group whose order is prime to the characteristic of the field and is therefore semi-simple. Then $U$ is irreducible over $A^{\prime}$, and by Curtis and Reiner (1962), Theorem 53.16, we have $\operatorname{dim}_{k} U^{\prime} \mid[S: R]$, hence $\operatorname{dim}_{k} U^{\prime}$ is prime to $p$. (Theorem 53.16 is proved in Curtis and Reiner (1962) when $k$ is the field of complex numbers, but is valid for any algebraically closed field of characteristic prime to the group order.) Now by (2),

$$
\left(\operatorname{dim}_{k} U\right)_{p}=|R|_{p}[H: S]_{p}=|H|_{p}
$$

since $S / R$ being a $p^{\prime}$-group implies that $|R|_{p}=|S|_{p}$. 
Returning to the calculation of $\left(\operatorname{dim}_{k} M_{p}\right)$, we have from (1) that

$$
\left(\operatorname{dim}_{k} M\right)_{p}=[T: V]_{p}[G: T]_{p}=|V|_{p}
$$

since $\left(\operatorname{dim}_{k} X\right)_{p}=1$ by hypothesis.

We now drop the assumption that $V$ is normal in $G$. Set $N=N_{G}(V)$. By the Green correspondence (Green (1964), Theorem 2), there exists an indecomposable $k N$-module $L$, with vertex $V$ and source $X$, such that

$$
L^{G}=M \oplus \sum_{i=1}^{m} L_{i}
$$

where each $L_{i}$ is an indecomposable $k G$-module with vertex $V_{i}$ conjugate to a subgroup of $V \cap V^{g_{i}}$ for some $g_{i} \in G-N$. Thus $\left|V_{i}\right|<|V|$, so the fact that $\left[G: V_{i}\right]_{p}$ divides $\operatorname{dim}_{k} L_{i}$ implies that

$$
[G: V]_{p}<\left(\operatorname{dim}_{k} L_{i}\right)_{p}, \quad 1 \leqslant i \leqslant m .
$$

Now the $k N$-module $L$ has vertex $V$ which is normal in $N$, and the source of $L$ has dimension prime to $p$, so we have proved above that $\left(\operatorname{dim}_{k} L\right)_{p}=[N: V]_{p}$. Since $\left(\operatorname{dim}_{k} L^{G}\right)_{p}=[G: N]_{p}\left(\operatorname{dim}_{k} L\right)_{p}$, we have

$$
\left(\operatorname{dim}_{k} L^{G}\right)_{p}=[G: V]_{p} .
$$

Taking the dimensions of both sides of (3) and applying (4) and (5), we have $\left(\operatorname{dim}_{k} M\right)_{p}=[G: V]_{p}$, proving the theorem.

COROLlary. Let $M$ be an indecomposable $k G$-module with cyclic vertex $V$, such that $N_{G}(V)$ is p-solvable. Then $\left(\operatorname{dim}_{k} M\right)_{p}=[G: V]_{p}$.

Proof. Let $X$ be a source of $M$. Since $V$ is cyclic, the indecomposable $k V$ modules are well known: there is (up to isomorphism) precisely one indecomposable $k V$-module of dimension $n$, for $1 \leqslant n \leqslant|V|$. Suppose that $\operatorname{dim}_{k} X=p m$; let $V_{1}$ be the subgroup of $V$ of index $p$, and let $X_{1}$ be the indecomposable $k V_{1}$-module of dimension $m$. Then $X$ and $X_{1}^{V}$ are both indecomposable $k V$-modules of dimension $p m$, hence are isomorphic. However, $X$ has vertex $V$, so $X$ cannot be an induced module. We conclude that $p \nmid \operatorname{dim}_{k} X$, and the corollary follows from the theorem.

Remark. These results need not hold if $N_{G}(V)$ is not $p$-solvable. Let $G$ be the symmetric group on 5 letters, and let $k$ have characteristic 3 . There is an indecomposable summand $M$ of $k G$ with $\operatorname{dim}_{k} M=9$ but $M$ has vertex 1 and trivial source, and $[G: 1]_{3}=3$. 


\section{References}

S. B. Conlon (1964), 'Twisted group algebras and their representations', J. Austral. Math. Soc. 4, 152-173.

C. W. Curtis and I. Reiner (1962), Representation theory of finite groups and associative algebras (Interscience, New York).

J. A. Green (1959), 'On the indecomposable representations of a finite group', Math. Z. 70, $430-445$.

J. A. Green (1964), 'A transfer theorem for modular representations', J. Algebra 1, 73-84.

P. A. Tucker (1965), 'On the reduction of induced indecomposable representations', Amer. J. Math. 87, 798-806.

Department of Mathematics

University of Alberta

Edmonton, Alberta

Canada 